\title{
Reliable overlapping control for civil structures
}

\author{
Lubomír Bakule ${ }^{a}$, Martin Papík and Branislav Rehák \\ Institute of Information Theory and Automation, Czech Academy of Sciences, CZ-182 08 Prague 8, Czech Republic
}

\begin{abstract}
The article presents results of experimental analysis targeted to actuator and sensor failures in the overlapping decentralized LQG feedback-based structure to mitigate responses of an earthquake-excited 20-story building. The building structure belongs to the second generation of civil engineering benchmark models. It includes a complete physical description, a high-fidelity finite element model and a Matlab/Simulink simulation framework. The presented performance analysis is focused on sensor and actuator failures appearing in individual local loops within two overlapped subsystems of an in-plane (2D) structure under the four real world historical earthquake records. The benchmark evaluation criteria and dynamic responses are analyzed to assess the acceptable performance.
\end{abstract}

\section{Introduction}

Large scale systems require control laws whose computation is efficient, and whose operation and implementation entails a minimum amount of information exchange amount the subsystems. It is well known that it leads to the development of the theory synthesizing control laws under decentralized information structure constraints [1]-[5].

Control of civil structures represents a new, difficult and unique problem, with many complexities in the processes of modeling, control design and implementation [6]-[11]. However, most structural control strategies are centralized, i.e. system output data collected by all senzors are fed into the centralized controller and sent to all actuators in a centralized manner. It is difficult to transmit huge amount of data between a set of distributed sensors and a central controller as well as to design such a controller. Moreover, if the centralized controller fails, the operation of the overall system can be essentially disrupted. Decentralized control, system decompositions and model simplifications were developed to overcome these difficulties.

Benchmark structural models have been proposed and tested as challenging problems to structural control community to design and compare control schemes for civil structures subjected to strong wind or earthquake excitations [9], [12]-[18]. Decentralized control strategies for building structures have been studied in [19]-[25].

The paper presents the results of experimental performance-based analysis focused on actuator and sensors failures in feedback control of the ASCE 20-story steel building benchmark proposed in [9]. It supposes availability of the overlapping decentralized LQG

\footnotetext{
${ }^{\mathrm{a}}$ Corresponding author: bakule@utia.cas.cz
}

controller satisfying the performance defined by 16 benchmark evaluation criteria and dynamic responses. Two overlapping subsystems are considered. The lower substructure is composed of floors 1-12, while the upper substructure is composed of floors 8-20. The overlapping appears in the part of the columns between the 8th and the 12th floors. The proposed structure has 6 sensors and 40 actuators. Proposed experimental analysis attempts to identify maximal possible sets of actuator and sensor failures in individual local feedback loops which satisfy the benchmark performance requirements. The sets of failed actuators and failed sensors are considered separately.

Note that, up to the author's knowledge, experimental analysis focused on the reliability ofsensors and actuators in the decentralized overlapping LQG controller implemented in civil structures to mitigate responses of an earthquake-excited has not yet been addressed.

\section{Problem}

The goal is to present experimental analysis targeted to actuator and sensor failures for the 20-story benchmark building structure with two overlapped subsystems by using decentralized LQG controllers to mitigate responses on the earthquakes. A complete physical description of the building benchmark problem, i.e. inplane (2D) finite element model and Matlab/Simulink simulation framework, performance evaluation criteria including a sample example, is available in [9]. The input excitation of the building structure is supposed to be one of the four real world historical earthquake records: (E1) El Centro (1940), (E2) Hachinohe (1968), (E3) Northridge (1994), and (E4) Kobe (1995). The N-S component of each earthquake record is used as the 
model input. Each proposed control strategy is evaluated for all earthquake records.

\subsection{The Problem}

The Problem is formulated as follows:

1. Propose operating number of sensors and actuators including their locations on the floors. Propose the overlapping decomposition of the structure and design a decentralized overlapping LQG controller for appropriately reduced order subsystems. Perform simulations to assess the dynamic behavior of the closedloop system including the performance evaluation.

2. Suppose availability of the decentralized overlapping LQG controller implemented in the bulding structure as proposed in the step 1. Analyze and experimentally identify sets of possible maximal numbers and locations of failed actuators (hydraulic dampers) and sensors (accelerometers) in each local feedback loop of the feedback system operatiing with acceptable performance. It includes the calculation and evaluation of evaluation criteria, analyzing responses and natural frequencies for all benchmark earthquake excitations.

\section{The approach for solving the problem}

The approach to solve the problem follows two subsequent steps. First, an appropriate controller must be proposed. Second, using such an implemented controller, possible sets of actuator failures as well as sensor failures in individual local loops are experimentally identified. It supposes that any tested set of failed actuators or sensors appears in one loop while the remaining loop operates without any failures. It means that the performance requirements must be satisfied to include such a set into acceptable sets. Actuator or sensor failures can be tested only experimentally because the performance criteria are primarily benchmark criteria focused on displacement, drift, acceleration, actuator saturation as well as dynamic responses. No exact reliable control design method enables such an evaluation directly.

Suppose that an appropriate controller is available. It is necessary to know the details of such a controller design to enable the subsequent experimental tests of acceptable failure sets. Such a controlled building model serves also as a reference case. The meaning of the performance criteria $J_{1-} J_{16}$ is explained in Appendix. Details of the controller design are surveyed as follows:

"The building structure is decomposed into two overlapping subsystems. The lower subsystem is composed of floors 1-12, while the upper subsystem is composed of floors 8-20. The overlapping appears in the part of the columns between the 8th and the 12th floors. The original mass and stiffness matrices have the order of 540 with two block diagonal blocks of the order 270 . These matrices are reduced to 526 DOFs by excluding the elements which are firmly attached to the ground. The matrices describing a lower subsystem $\mathrm{S} 1$ are reduced to 256 DOFs. The matrices describing an upper subsystem
S2 are not reduced, i.e. their dimensions remain unchanged. Then, the Ritz and Guyan reductions follow. It results in a reduced mass and stiffness matrices of order 135 with a block diagonal structure where the lower and the upper blocks have the dimensions 63 and 72. The corresponding state-space system has the dimension 270 . The subsequent model reduction results in the systems denoted S1R and S2R of the dimensions 32 and 30, respectively. Suppose the sensor models are identical with those used in the sample control design example, but their location and number are different from the sample example. They are located on floors $2,4,8,14,18$ and the roof. Hydraulic actuators are selected identically with those ones used in the sample example [9]. It means that the dynamics of the actuators is modelled with a capacity of $897 \mathrm{kN}$. However, their location and number is also changed. Sensors and actuators appear also in the interconnection, i.e. between the 8th and the 12th floors. A total of 40 actuators are used. The numbers of actuators and their location on the floors are based on the analysis of physical properties, the decomposed overlapping structure and simulations. The sequence of these numbers is from the bottom to the roof as $2,1,1,1,1,1,1,1,1,2,3,4,4,4,3,2,1,1,3,3$. It remains to add 20 equations of the actuators which are divided as 12 and 13 for the subsystems S1R and S2R, respectively. Therefore, the closed-loop reduced-order control design system SRC has the dimension 87 with the local closedloop subsystem dimensions of order 44 and 43 . Recall that the resulting controller gain matrix $\mathrm{K}$ has the dimensions $20 \times 87$. It is composed of the block matrices $\mathrm{K} 1$ and $\mathrm{K} 2$ of dimensions $12 \times 43$ and $13 \times 44$, respectively. The resulting observer gain matrix $\mathrm{L}$ has the dimensions $87 \times 6$. It is composed of the block matrices L1 and L2 of dimensions $44 \times 3$ and $43 \times 3$, respectively. A decentralized control law is proposed for each free subsystem by combining its model reduction and the LQG design on the reduced order subsystems.

Table 1. No failures - Criteria (left), natural frequencies (right)

\begin{tabular}{|c|c|c|}
\hline & Pre & Post \\
\hline \hline$J_{1}$ & 0.9001 & 0.9582 \\
\hline$J_{2}$ & 0.8757 & 0.9994 \\
\hline$J_{3}$ & 0.9637 & 0.9982 \\
\hline$J_{4}$ & 0.9348 & 1.1039 \\
\hline$J_{5}$ & 0.7614 & 0.7341 \\
\hline$J_{6}$ & 0.7517 & 0.6712 \\
\hline$J_{7}$ & 0.6979 & 0.7398 \\
\hline$J_{8}$ & 0.7539 & 0.6780 \\
\hline$J_{9}$ & 0.0134 & 0.0114 \\
\hline$J_{10}$ & 0.0884 & 0.1006 \\
\hline$J_{11}$ & 0.0171 & 0.0153 \\
\hline$J_{12}$ & 0.0377 & 0.0341 \\
\hline$J_{13}$ & 40 & 40 \\
\hline$J_{14}$ & 6 & 6 \\
\hline$J_{15}$ & 87 & 87 \\
\hline$J_{16}$ & 730.29 & 617.73 \\
\hline
\end{tabular}




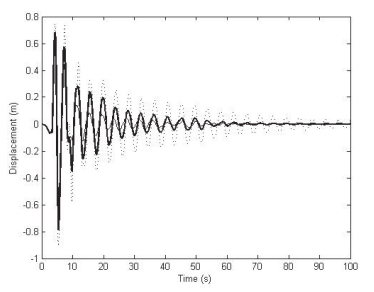

a) Displacement

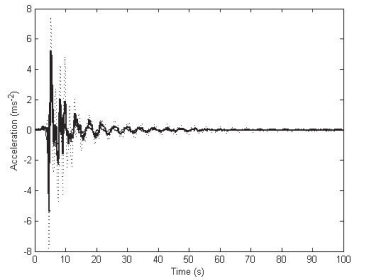

c) Acceleration

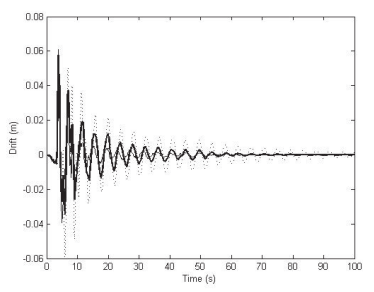

b) Drift

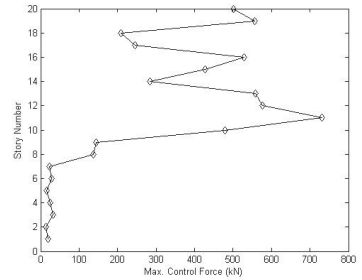

d) Max. Control Force

Figure 1. No failures - Pre-earthquake model

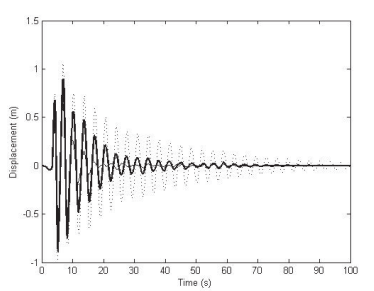

a) Displacement

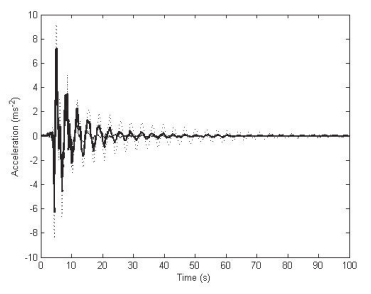

c) Acceleration

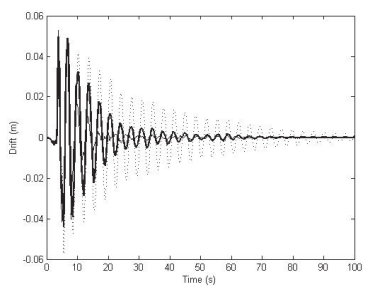

b) Drift

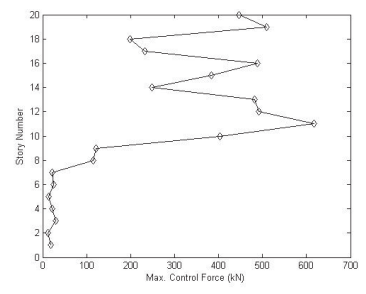

d) Max. Control Force
Figure 2. No failures - Post-earthquake model
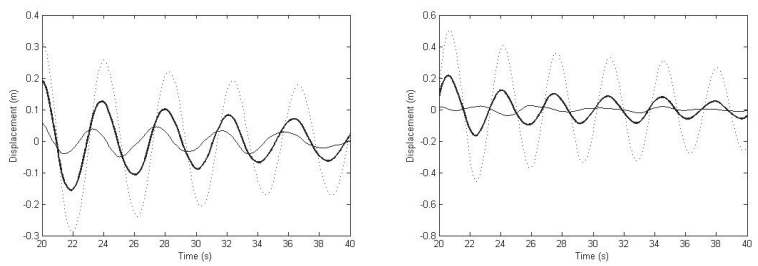

a) Displacement
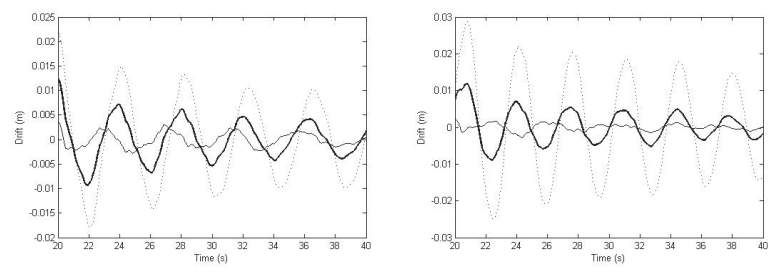

b) Drift
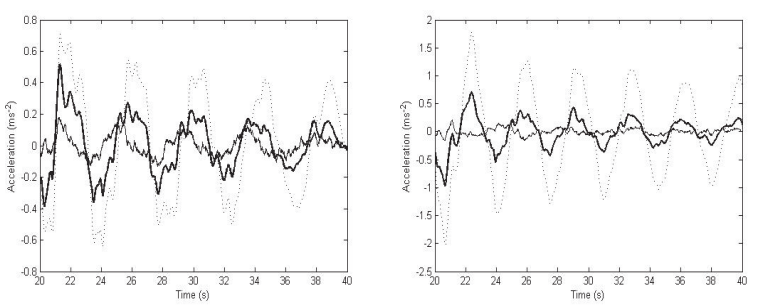

c) Acceleration

Figure 3. No failures: Pre- (left), Post- (right)
The values of the performance criteria are surveyed. Tables present maximal values of the performance criteria over all four earthquakes. 10 natural frequencies are given. Figures display the responses (Bold) to the Northridge earthquake record and the responses (Solid) of centralized sample example by [9] for the preearthquake and the post-earthquake models. The openloop system responses are included (Dotted). The 20th floor displacement and acceleration as well as the 2nd floor drift responses are displayed on all figures", as summarized in [18].

\section{The results}

The results are divided into two groups according to the type of failure in each local loop: actuator failures and sensor failures. Partial failures in \% are acceptable. For instance, the set of actuator failures in the lower subsystem supposes no failures in sensors of both subsystems and no failure in actuators in the upper subsystem. It means the sets of actuator and sensor failures are separated according to individual loops. The sets of actuators or sensors are also considered separately.

Tables present maximal numbers and locations of acceptable failures of actuators and sensors and the responses of the structure under actuator failures. The responses of sensor failures are similar, therefore they are omitted. The values of the performance criteria over all four earthquakes are given. Figures display the responses (Bold) to the Northridge earthquake record and the responses (Solid) of the system by [20] for the preearthquake and the post-earthquake models. The openloop system responses are included (Dotted). The 20th floor displacement and acceleration and the 2nd floor drift responses are displayed on all figures.

\subsection{Failures in the lower subsystem}

Table 2. Locations of failed actuators (left) and sensors (right)

\begin{tabular}{|c|c|c|}
\hline Floor & No F & Lower F \\
\hline \hline 1 & 2 & 2 \\
\hline 2 & 1 & 0 \\
\hline 3 & 1 & 0 \\
\hline 4 & 1 & 0 \\
\hline 5 & 1 & 0 \\
\hline 6 & 1 & 0 \\
\hline 7 & 1 & 0 \\
\hline 8 & 1 & $1(40 \%)$ \\
\hline 9 & 1 & $1(10 \%)$ \\
\hline 10 & 2 & 1 \\
\hline 11 & 3 & 1 \\
\hline 12 & 4 & 1 \\
\hline 13 & 4 & 4 \\
\hline 14 & 4 & 4 \\
\hline 15 & 3 & 3 \\
\hline 16 & 2 & 2 \\
\hline 17 & 1 & 1 \\
\hline 18 & 1 & 1 \\
\hline 19 & 3 & 3 \\
\hline 20 & 3 & 3 \\
\hline & & \\
\hline
\end{tabular}

\begin{tabular}{|c|c|c|}
\hline Floor & No $\mathrm{F}$ & Lower $\mathrm{F}$ \\
\hline \hline 2 & 1 & 0 \\
\hline 4 & 1 & 0 \\
\hline 8 & 1 & $1(15 \%)$ \\
\hline 14 & 1 & 1 \\
\hline 18 & 1 & 1 \\
\hline 20 & 1 & 1 \\
\hline
\end{tabular}


Table 3. Failures of actuators - Criteria

\begin{tabular}{|c|c|c|}
\hline & Pre & Post \\
\hline \hline$J_{1}$ & 0.9371 & 0.9739 \\
\hline$J_{2}$ & 0.9027 & 0.9824 \\
\hline$J_{3}$ & 0.9537 & 0.998 \\
\hline$J_{4}$ & 0.9554 & 1.0668 \\
\hline$J_{5}$ & 0.8205 & 0.806 \\
\hline$J_{6}$ & 0.8108 & 0.7525 \\
\hline$J_{7}$ & 0.7834 & 0.7974 \\
\hline$J_{8}$ & 0.8158 & 0.7577 \\
\hline$J_{9}$ & 0.0106 & 0.0094 \\
\hline$J_{10}$ & 0.0999 & 0.0988 \\
\hline$J_{11}$ & 0.0127 & 0.0118 \\
\hline$J_{12}$ & 0.0274 & 0.0245 \\
\hline$J_{13}$ & 40 & 40 \\
\hline$J_{14}$ & 6 & 6 \\
\hline$J_{15}$ & 87 & 87 \\
\hline$J_{16}$ & 575.14 & 509.63 \\
\hline
\end{tabular}

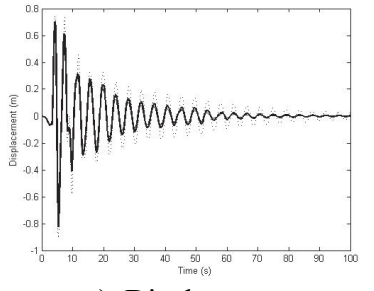

a) Displacement

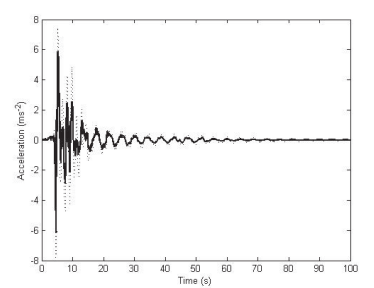

c) Acceleration

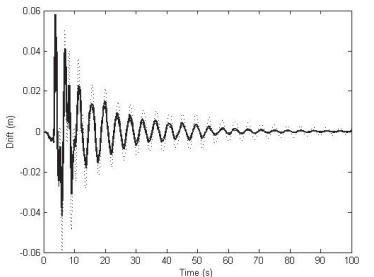

b) Drift

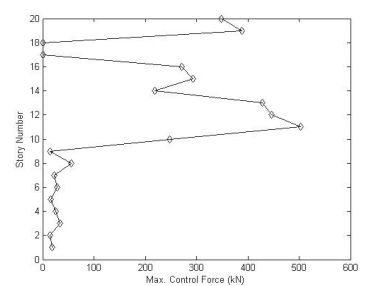

d) Max. Control Force
Figure 4. Failures in upper part - Pre-earthquake model

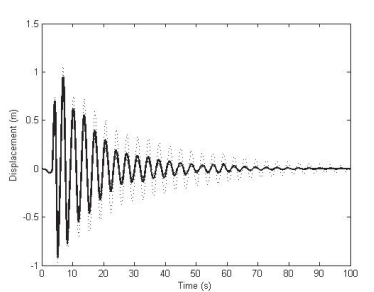

a) Displacement

c) Acceleration

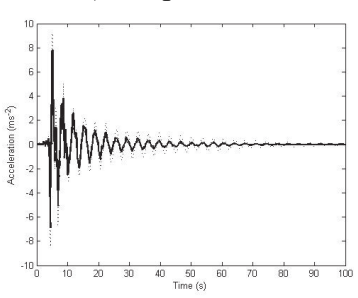

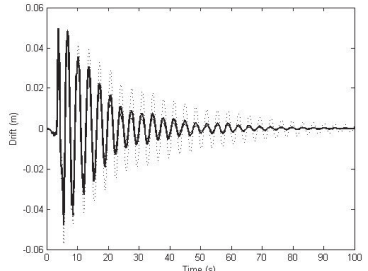

b) Drift

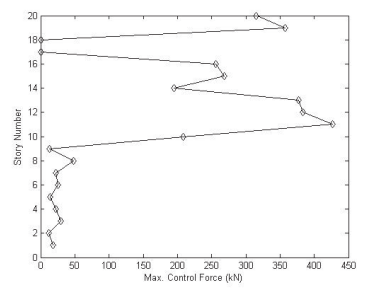

d) Max. Control Force
Figure 5. Failures in upper part - Post-earthquake model
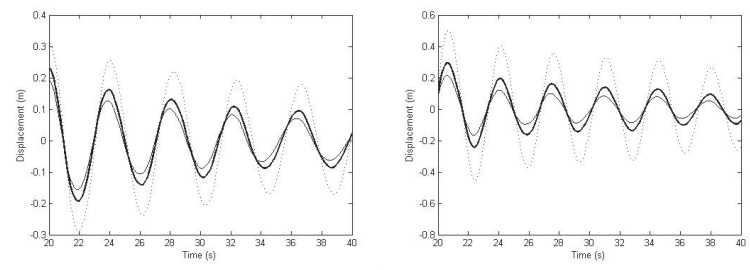

a) Displacement
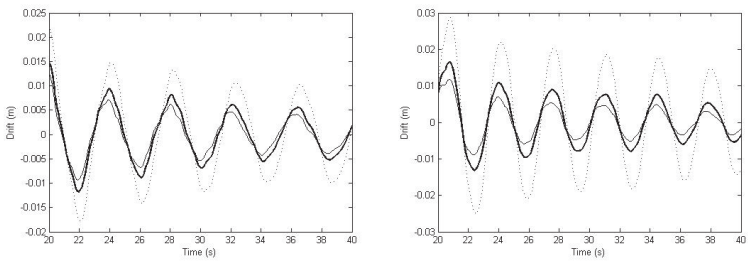

b) Drift

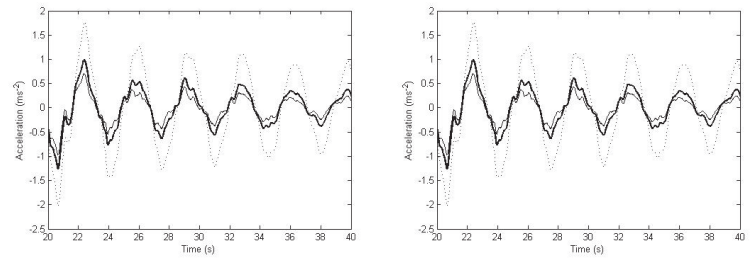

c) Acceleration

Figure 6. Failures in upper part: Pre- (left), Post- (right)

\subsection{Failures in the upper subsystem}

Note only that the presented structure of tables and figures fully corresponds with their meaning and structure shown in the previous subsection 4.1. The results of numerical experiments are given for both the pre-earthquake model and the post-earthquake model.

Table 4. Locations of failed actuators (left) and sensors (right)

\begin{tabular}{|c|c|c|}
\hline Floor & No F & Upper F \\
\hline \hline 1 & 2 & 2 \\
\hline 2 & 1 & 1 \\
\hline 3 & 1 & 1 \\
\hline 4 & 1 & 1 \\
\hline 5 & 1 & 1 \\
\hline 6 & 1 & 1 \\
\hline 7 & 1 & 1 \\
\hline 8 & 1 & $1(40 \%)$ \\
\hline 9 & 1 & $1(10 \%)$ \\
\hline 10 & 2 & 1 \\
\hline 11 & 3 & 2 \\
\hline 12 & 4 & 3 \\
\hline 13 & 4 & 3 \\
\hline 14 & 4 & 2 \\
\hline 15 & 3 & 1 \\
\hline 16 & 2 & 0 \\
\hline 17 & 1 & 0 \\
\hline 18 & 1 & 0 \\
\hline 19 & 3 & 2 \\
\hline 20 & 3 & 2 \\
\hline & & \\
\hline
\end{tabular}


Table 5. Failures of actuators in the lower subsystem - Criteria

\begin{tabular}{|c|c|c|}
\hline & Pre & Post \\
\hline \hline$J_{1}$ & 0.9137 & 0.9654 \\
\hline$J_{2}$ & 0.883 & 0.9986 \\
\hline$J_{3}$ & 0.9518 & 0.9977 \\
\hline$J_{4}$ & 0.9302 & 1.0849 \\
\hline$J_{5}$ & 0.7824 & 0.7592 \\
\hline$J_{6}$ & 0.7708 & 0.7004 \\
\hline$J_{7}$ & 0.7256 & 0.7482 \\
\hline$J_{8}$ & 0.7726 & 0.7063 \\
\hline$J_{9}$ & 0.0104 & 0.0094 \\
\hline$J_{10}$ & 0.0925 & 0.1005 \\
\hline$J_{11}$ & 0.0152 & 0.0145 \\
\hline$J_{12}$ & 0.0347 & 0.0312 \\
\hline$J_{13}$ & 40 & 40 \\
\hline$J_{14}$ & 6 & 6 \\
\hline$J_{15}$ & 87 & 87 \\
\hline$J_{16}$ & 562.94 & 512.37 \\
\hline
\end{tabular}

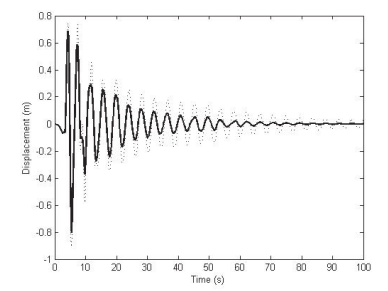

a) Displacement

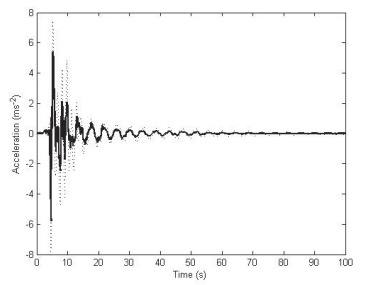

c) Acceleration

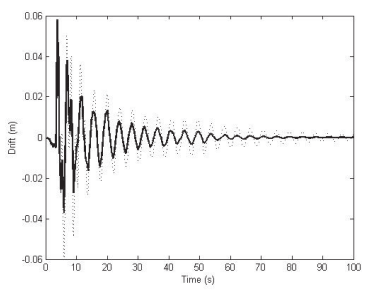

b) Drift

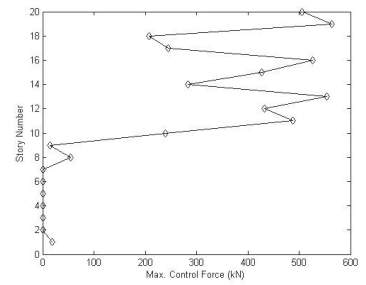

d) Max. Control Force
Figure 7. Failures in lower part - Pre-earthquake model

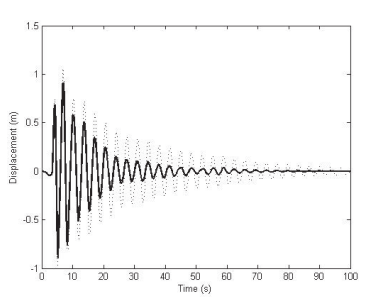

a) Displacement

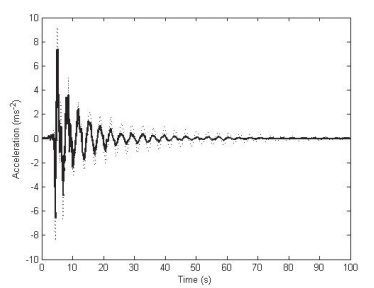

c) Acceleration

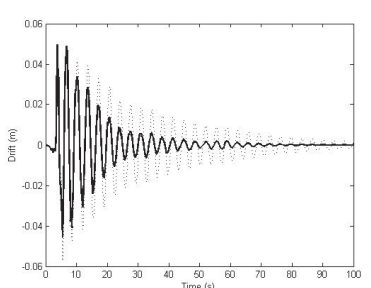

b) Drift

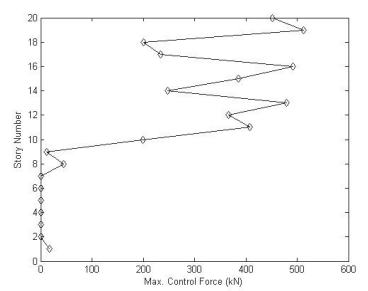

d) Max. Control Force
Figure 8. Failures in lower part - Post-earthquake model
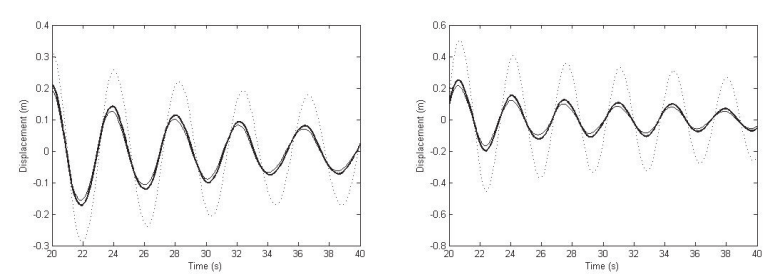

a) Displacement
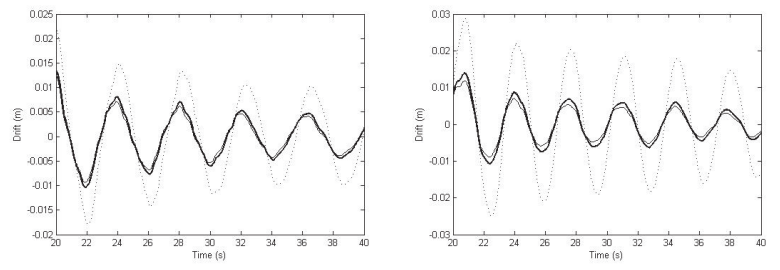

b) Drift

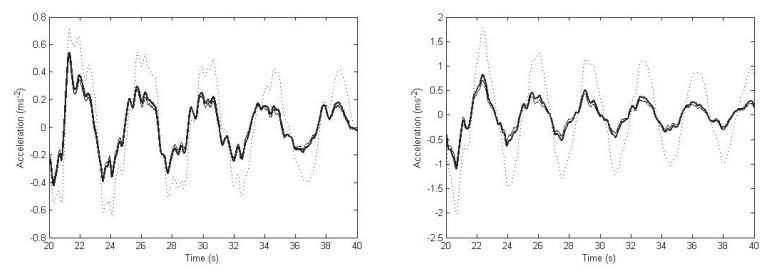

c) Acceleration

Figure 9. Failures in lower part: Pre- (left), Post- (right).

\section{Conclusion}

The paper contributes by the methodology of experimental analysis and tests for the decentralized overlapping reliable LQG design focused on the 20-story in-plane (2-D) benchmark high-fidelity building FEM model. Performance of the closed-loop system under sensors and actuators failures has been tested and evaluated using numerical experiments. The tests show that the presented approach yields sufficient reliability of actuators and sensors in individual local feedback loops. It offers a variety of possible extensions in various decomposition approaches for civil structures and applied control design methods.

\section{Acknowledgement}

The authors are grateful to the Czech Science Foundation by its support through the Grant 13-02149S.

\section{Appendix}

Appendix surveys the applied performance criteria including the constraints of the device which are used in the numerical experiments. Denote a set of all four used real world historical earthquake records $E$.

"A systematic evaluation of the performance is based on the evaluation criteria $J_{1}-J_{16}$. The criteria $J_{1}-J_{15}$ are those used by [9]. The criterion $J_{16}$ has been added. It is the value of a maximal actuator force corresponding with the current simulation run. It is required to keep this value less than the capacity of $897 \mathrm{kN}$ which is allowed for hydraulic actuators. The criteria $J_{1}-J_{3}$ have been selected as the most significant criteria. More precisely, these criteria are defined as follows 


$$
J_{1}=\max _{E}\left(\frac{\max _{t, i}\left|x_{i}(t)\right|}{x^{\max }}\right)
$$

where $J_{1}$ denotes the maximum displacement over the set of all states $x_{\mathrm{i}}(t)$ corresponding to the horizontal displacement of floors relative to the ground. $x^{\max }$ is the maximum uncontrolled displacement corresponding to each respective earthquake.

$$
J_{2}=\max _{E}\left(\frac{\max _{t, i}\left|d_{i}(t)\right|}{d^{\max }}\right)
$$

where $J_{2}$ denotes the maximum inter-story drift over the set of all states $x_{\mathrm{i}}(t)$ corresponding to the drift of floors. $d^{\max }$ is the maximum inter-story drift corresponding to each respective earthquake.

$$
J_{3}=\max _{E}\left(\frac{\max _{t, i}\left|\ddot{x}_{a i}(t)\right|}{\ddot{x}_{a}^{\max }}\right)
$$

where $J_{3}$ denotes the maximum floor acceleration corresponding to the drift of floors. $\ddot{x}_{\mathrm{a}}{ }^{\text {max }}$ is the maximum uncontrolled floor acceleration corresponding to each respective earthquake.

A short summary of the evaluation criteria follows:

$$
\begin{aligned}
& J_{1} \text { - Floor displacement } \\
& J_{2} \text { - Inter-story drift } \\
& J_{3} \text { - Floor acceleration } \\
& J_{4} \text { - Base shear } \\
& J_{5} \text { - Normed floor displacement } \\
& J_{6} \text { - Normed inter-story drift } \\
& J_{7} \text { - Normed floor acceleration } \\
& J_{8} \text { - Normed base shear } \\
& J_{9} \text { - Control force } \\
& J_{10} \text { - Control device stroke } \\
& J_{11} \text { - Control power } \\
& J_{12} \text { - Normed control power } \\
& J_{13} \text { - Control devices } \\
& J_{14} \text { - Sensors } \\
& J_{15} \text { - Computational resources } \\
& J_{16} \text { - Maximum actuator force }
\end{aligned}
$$

Note that the values of the criteria $J_{1-} J_{8}$ are equal to one, while the values of the remaining criteria are equal to zero for the uncontrolled system. Any successful controller design corresponds with the values of the criteria $J_{1}-J_{8}$ less than one. The post-earthquake model has decreased stiffness caused by assumed structural damages compared with the pre-earthquake model. Simulations have shown that the usage of the postearthquake model for the control design with a subsequent verification on the closed-loop system composed of the pre-earthquake model with the feedback gain matrices generated for the post-earthquake model is more convenient approach than the usage of the models in an opposite order. Therefore, the proper decentralized LQG design has been performed for the post-earthquake model as the case corresponding with the worst possible scenario", as summarized in [17].

The solved problem employs hydraulic actuators with a capacity of $897 \mathrm{kN}$, a stroke of $\pm 8.9 \mathrm{~cm}$ and the control signal as required in [9].

\section{References}

1. D.D. Šiljak, Decentralized Control of Complex Systems, (Academic Press, NY, 1991)

2. L. Bakule, Annual Reviews in Control, 32 ,87-98, (2008)

3. M.S. Mahmoud, Decentralized Control and Filtering in Interconnected Dynamical Systéme, (CRC Press, Boca Raton, 2011)

4. L. Bakule, M. Papík, Annual Reviews in Control, 36, 1, 1-10 (2012)

5. L. Bakule, Annual Reviews in Control, 38, 1, 71-80 (2014)

6. G. S. West-Vukovich, E. D. Davison, P. C Huges., IEEE T Automat Contr, 29 (10):866-879, 1984.

7. S.M. Joshi, Control of Large Flexible Space Structures, (Springer, NY, 1989)

8. G.W. Housner, L. A. Bergman, T. K. Caughey, A. G. Chassiakos, R. O. Claus, S. F.Masri, R. E. Skelton, T. T. Soong, B. F. Spencer, J. T. P. Yao, J Eng Mech-ASCE, 123, 9, 897-971, (1997)

9. B.F. Spencer Jr., R. Christenson, S.J. Dyke, Proceedings of the Second World Conference on Structural Control, 1351-1360 (1998)

10. W.K. Gawronski., Advanced Structural Dynamics and Active Control of Structures, (Springer, NY 2004)

11. A. Preumont, Vibration Control of Active Structures. An Introduction, (Springer, Berlin Heidelberg, 2011)

12. S.J. Dyke, J.M. Caicedo, G. Turan, L.A. Bergman, S. Hague, J Struct Eng-ASCE, 129, 7, 857-872 (2003)

13. A. K. Agrawall, J. N. Yang, W. L. He, J Struct EngASCE 129, 7, 884-894, (2003)

14. T.R. Alt, F. Jabbari, J.N. Yang, Earthquake Eng Struc, 29, 241-257 (2000)

15. L. Bakule, F. Paulet-Crainiceanu, J. Rodellar, J.M. Rossell, IEEE T Contr Syst T, 13, 4, 663-669 ( 2005)

16. L. Bakule, M. Papík, B. Rehák, Proceedings of the 19th World Congress of the IFAC, 10427-10432 (2014)

17. L. Bakule, M. Papík, B. Rehák, Proceedings of the 6th World Conference on Structural Control and Monitoring, 2242-2253 ( 2014)

18. L. Bakule, M. Papík, B. Rehák, Proceedings of the 7th ECCOMAS Thematic Conference on Smart Structures and Materials, 1-15 (2015)

19. L. Bakule, J. Rodellar, International Journal of Control, 61, 3, 559-570 (1995)

20. L. Bakule, J. Rodellar., International Journal of Control, 61, 3, 571-587, (1995)

21. Y. Wang, J.P. Lynch, K.H. Law, Earthquake Eng Struc, 38, 377-401 (2009)

22. Y. Lei, D.T. Wu, Y. Lin, Computer-Aided Civil and Infrastructure Engineering, 27, 2-13,(2012)

23. Y. Lei, D.T. Wu, Procedia Engineering, 14, 1229$1236(2011)$

24. Y. Lei, D.T. Wu, S.-Z. Lin, Eng Struct, 52, 306-316, (2013)

25. H. Li, J. Wang, G. Song, L.Y. Li, Structural Control and Health Monitoring, 18, 2, 227-240 (2011) 